\title{
高氢渗透分离性的沸石杂化支撑炭膜的制备
}

\author{
张 兵 $^{1}$, 江 园 ${ }^{1}$, 吴永红 ${ }^{1}$ ，鲁云华 ${ }^{2}$, 赵丹丹 ${ }^{1}$ ，王同华 ${ }^{3}$ \\ (1. 沈阳工业大学 石油化工学院, 辽阳 111003; 2. 辽宁科技大学 功能材料省级重点实验室, 鞍山 114051; 3. 大连 \\ 理工大学 精细化工国家重点实验室, 大连 116024)
}

摘 要: 以 ZSM-5 沸石掺杂改性的 1, 4-双(4-氨基-2-三氟甲基-苯氧基)苯-1, 2, 3, 4-环丁烷四甲酸二酐型聚酰亚胺为 前驱体，通过旋涂成膜和热解过程制备了平板状支撑炭膜。采用热失重、红外光谱、X 射线衍射、扫描电镜及气体 渗透技术分别研究了前驱体热稳定性、膜表面官能团、微结构, 微观形貌及分离性能。考察了 ZSM-5 掺杂量及热 解温度对炭膜结构和气体分离性的影响。结果表明: 经 ZSM-5 改性后前驱体热稳定性与残炭量降低, 炭膜微观结 构变致密; 加入沸石显著提高了炭膜的渗透性, 且随 ZSM-5 掺杂量增加, 气体渗透性先减小后增大; 随着热解温度升高, 炭膜的渗透性与选择性皆减小。经 $650^{\circ} \mathrm{C}$ 热解制得杂化炭膜对 $\mathrm{H}_{2} / \mathrm{N}_{2}$ 体系的分离性能均远超过 Robeson 上界限。

关 键 词: 聚酰亚胺; 沸石; 炭膜; 分离性能

中图分类号: TQ028 文献标识码: A

\section{Fabrication of Zeolite Hybrid Supported Carbon Membranes with High Hydrogen Permselective Performance}

\author{
ZHANG Bing $^{1}$, JIANG Yuan ${ }^{1}$, WU Yong-Hong ${ }^{1}$, LU Yun-Hua ${ }^{2}$, ZHAO Dan-Dan ${ }^{1}$, WANG Tong-Hua ${ }^{3}$ \\ (1. School of Petrochemical Engineering, Shenyang University of Technology, Liaoyang 111003, China; 2. Provincial Key \\ Laboratory of Functional Materials, University of Science and Technology Liaoning, Anshan 114051, China; 3. State Key \\ Laboratory of Fine Chemical Engineering, Dalian University of Technology, Dalian 116024, China)
}

\begin{abstract}
Plate supported carbon membranes were prepared by precursor of 1, 4-bis(4-amino-2-trifluoromethylphenoxy) benzene-1, 2, 3, 4-cyclobutanetetracar-boxylic dianhydride type polyimide modified with zeolite ZSM-5, through the processes of spin-coating and pyrolysis. Thermal stability of precursor, surface functional groups, microstructure, morphology, and separation performance of membranes were characterized by the techniques of thermogravimetric analysis, infrared spectroscopy, X-ray diffraction, scanning electron microscope, and gas permeation, respectively. Effects of incorporating amount of ZSM-5 and pyrolysis temperature on structure and gas separation performance of carbon membranes were investigated. Results show that thermal stability and carbon residue of precursor are reduced by ZSM-5 modification. Simultaneously, the microstructure of carbon membranes becomes more compact. Incorporation of zeolite remarkably increases the gas permeability of carbon membranes. In addition, the gas permeability first decreases then increases with increased incorporation of ZSM-5. As the pyrolysis temperature elevating, both permeability and selectivity of as-obtained carbon membranes decrease. The separation performance
\end{abstract}

收稿日期：2015-08-31; 收到修改稿日期：2015-10-10

基金项目: 国家自然科学基金(20906063,21376037, 21436009,21406102), 国家 863 项目(2012AA03A611), 辽宁省自然科学 基金(20102170); 辽宁省高等学校杰出青年学者基金(LJQ2012010); 辽宁省功能材料重点实验室开放课题 (USTLKFSY201507)

National Natural Science Foundation of China (20906063, 21376037, 21436009, 21406102); 863 High-Tech Project of China (2012AA03A611); Liaoning Natural Science Foundation of China (20102170); Program for Liaoning Excellent Talents in University (LJQ2012010); Liaoning Key Laboratory of Functional Materials (USTLKFSY201507)

作者简介: 张 兵(1977-), 男，博士，教授. E-mail: zhangbing@sut.edu.cn

通讯作者:鲁云华，副教授.E-mail: lee.lyh@163.com; 王同华, 教授.E-mail: wangth@dlut.edu.cn 
performance of hybrid carbon membranes prepared at pyrolysis temperature of $650^{\circ} \mathrm{C}$ is by far the Robeson's upper bound for $\mathrm{H}_{2} / \mathrm{N}_{2}$ system.

Key words: polyimide; zeolite; carbon membranes; separation performance

氢气既是重要的工业原料, 又是极具吸引力的 清洁能源载体 ${ }^{[1]}$ 。随着整体煤气化联合循环发电系 统( IGCC)和燃料电池等先进技术对高纯度氢源要求 的不断提高, 氢气纯化已成为生产过程或使用前最 重要环节之一[2-3]。膜分离技术以其高效、环保、节 能等优势, 已迅速成长为氢气纯化的重要手段 ${ }^{[4]}$ 。

炭膜是一种新型膜材料, 具有优异分离性能, 以及耐热、耐腐蚀、抗污染等优势, 在许多领域突 显诱人前景 ${ }^{[5-8]}$ 。尽管如此, 为进一步提高炭膜的性 价比与市场竞争力, 仍需改善其分离性 ${ }^{[9]}$ 。然而, 在 炭膜制备过程中的热解阶段, 石墨化进程会导致炭 膜微观结构致密化，使渗透性的提升空间十分有 限 ${ }^{[10]}$ 。因此, 需掺杂易分解有机物或多孔无机物来 增大前驱体的刚性与自由体积分数, 或金属粒子优 先吸附特定气体，来达到提高炭膜渗透性目的 ${ }^{[10]}$ 。 其中, 有机物热稳定性差, 易在炭膜基体内形成大 孔，从而降低炭膜的制备可控性与分离性 ${ }^{[11]}$; 金属 粒子热稳定性虽好, 但其既无孔隙又对气体吸附力 微弱, 使渗透性改善效果不大 ${ }^{[12]}$ 。而如沸石等多孔 无机材料则可通过自身丰富孔隙有效地在炭膜基体 内构造大量渗透通道, 降低气体扩散阻力, 从而提 高渗透性 ${ }^{[13-15]}$ 。

由于炭膜是以分子篮分机理为主实现气体分离, 通常对分离 $\mathrm{O}_{2} / \mathrm{N}_{2}$ 体系较理想, 而对 IGCC 与燃料电 池领域所涉及的 $\mathrm{H}_{2} / \mathrm{N}_{2}$ 与 $\mathrm{H}_{2} / \mathrm{CO}_{2}$ 体系的分离选择性 则不佳。本工作尝试采用沸石对本课题组近期开发 的 1, 4-双(4-氨基-2-三氟甲基-苯氧基)苯-1，2，3，4环丁烷四甲酸二酕基聚酰亚胺(6FAPB-CBDA)型 聚酰亚胺进行掺杂改性, 以期获得高氢渗透选择 性炭膜。

\section{1 实验方法}

\section{1 实验原料}

前驱体为自制 6FAPB-CBDA 型聚酰亚胺的预 聚体聚酰胺酸在 N-N 二甲基乙酰胺中的溶液(固含 量为 15\%)。其它试剂有分析级六次甲基四胺(国药 集团化学试剂有限公司), 羧甲基纤维素(辽阳市彩 塔纤维素厂), 酚醛树脂(河南新乡伯马风帆实业有 限公司)及 ZSM-5 型沸石(大连理工大学, 平均粒径

\section{$1.25 \mu \mathrm{m})$ 。}

\section{2 支撑炭膜的制备}

首先, 按文献[16]所述方法, 在成型压力 $2 \mathrm{MPa}$ 下压制成型 $\phi 3 \mathrm{~cm} \times 0.4 \mathrm{~cm}$ 的圆片状支撑体。另外， 以聚酰胺酸溶液为基准，按其质量分数的 $0 \sim 0.5 \mathrm{wt} \%$ 添加 ZSM-5 沸石, 经适当搅拌、超声波分散与静置 脱泡, 配置成膜液。在支撑体表面滴加 $0.2 \mathrm{~g}$ 涂膜液, 放于旋转涂膜机上，以 $900 \mathrm{r} / \mathrm{min}$ 转速水平旋转 $30 \mathrm{~s}$, 室温下自然干燥 $5 \mathrm{~h}$ 后再次旋涂。依此重复涂膜-干 燥 6 次后, 自然干燥 $5 \mathrm{~d}$, 得到支撑聚合物膜。将聚 合物膜标记为 PMZ- $x$, “ $x$ ”代表 ZSM-5 质量分数 (0 0.5wt \%)。

最后, 将聚合物膜置于卧式炭化炉中进行热 解。在 $\mathrm{N}_{2}$ 流量 $200 \mathrm{~mL} / \mathrm{min}$ 保护下, 以 $2^{\circ} \mathrm{C} / \mathrm{min}$ 从室 温逐步升至 $100^{\circ} \mathrm{C} 、 200^{\circ} \mathrm{C} 、 300^{\circ} \mathrm{C} 、 400^{\circ} \mathrm{C}$ 并分别恒 温 $30 \mathrm{~min}$, 再以 $1^{\circ} \mathrm{C} / \mathrm{min}$ 升至终温 $\left(650^{\circ} \mathrm{C} 、 750^{\circ} \mathrm{C}\right.$ 、 $\left.850^{\circ} \mathrm{C}\right)$ 。将炭膜标记为 CMZ- $x-T$, “ $x$ ”代表 ZSM-5 掺 杂量 $(0 \sim 0.5 \mathrm{wt} \%)$, “ $T$ ”代表热解温度 $\left(650^{\circ} \mathrm{C} 、 750^{\circ} \mathrm{C}\right.$ 及 $\left.850^{\circ} \mathrm{C}\right)$ 。

\section{3 表征方法}

采用 TGA-4000 型热失重分析仪测试前驱体的 热稳定性，采用 Nicolet Nexus 470 型全反射红外光 谱仪检测样品表面官能团，采用 TM-3000 型扫描电 镜观察炭膜的微观形貌, 采用 RINT2000 型 X 射线 衍射仪对膜样品结构进行分析(电压 $40 \mathrm{kV}$, 电流 $100 \mathrm{~mA}$ )。另外, 根据 $\mathrm{X}$ 射线衍射角与 Bragg 公式 $d_{002}=\lambda /(2 \sin \theta)$ 得到微晶炭层间距 $d_{002}$ 值 ${ }^{[17]}$ 。采用传 统的恒压力变体积法测定了炭膜在 $0.1 \mathrm{MPa}$ 和 $30^{\circ} \mathrm{C}$ 时对高纯单组份气体的渗透性与选择性 ${ }^{[18]}$ 。

\section{2 结果与讨论}

\section{1 前驱体热稳定性}

图 1 所示为前驱体的热失重曲线 $(\mathrm{TG})$ 与失重速 率曲线(DTG)。所有前驱体的 TG 与 DTG 曲线形状 均相似, 且都包含两个主要失重阶段: $200 \sim 350^{\circ} \mathrm{C}$ 、 $400 \sim 700^{\circ} \mathrm{C}, 150^{\circ} \mathrm{C}$ 前失重由样品中少量残留水分和 溶剂挥发造成。第一阶段失重是由于聚酰胺酸分子 环化亚胺化脱水形成的; 第二阶段失重主要以热分 解为主, 随着大量官能团从主链上脱落, 析出 $\mathrm{CO}$ 、 

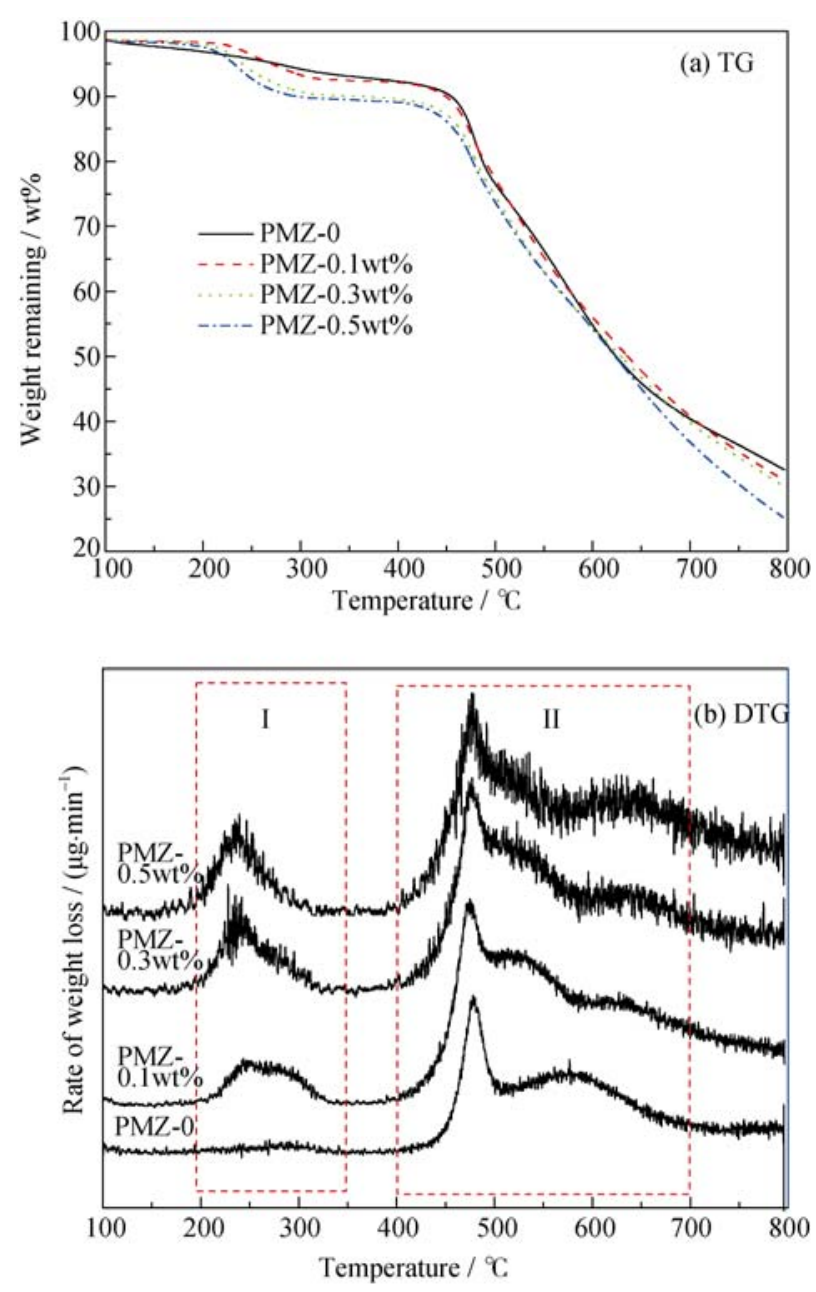

图 1 前驱体膜热分析曲线

Fig.1 Thermal analysis curves of precursors

$\mathrm{CO}_{2} 、 \mathrm{CH}_{4}$ 等气体。而 $700^{\circ} \mathrm{C}$ 以上分解反应变缓, 通 过芳环自由基重排形成类石墨化结构。

对于杂化膜, 由于 ZSM-5 合成时残留的有机模 板剂脱除及其酸性催化作用促进了分子链间(或内) 亚胺化反应衍生大量水, 从而使失重更明显、失重速 率峰强增大 ${ }^{[19]}$ 。在 $800^{\circ} \mathrm{C}$ 时, 前驱体膜的残炭量顺序 为 PMZ-0 $>$ PMZ-0.1wt $\%>$ PMZ- $0.3 w t \%>P M Z-0.5 w t \%$, 表明加入 ZSM-5 降低了前驱体的热稳定性。

\section{2 膜表面官能团分析}

图 2 是样品的红外光谱图, 从图 2 可以看出, 在 前驱体膜红外谱图中可见 $-\mathrm{C}=\mathrm{O}$ 不对称伸缩振动 $\left(1712 \mathrm{~cm}^{-1}\right) 、 \mathrm{C}-\mathrm{N}$ 伸缩振动 $\left(1374 \mathrm{~cm}^{-1}\right)$ 、芳醚的伸缩 振动 $\left(1230 \mathrm{~cm}^{-1}\right) 、-\mathrm{CF}_{3}$ 中 $\mathrm{C}-\mathrm{F}$ 伸缩振动 $\left(1160 \mathrm{~cm}^{-1}\right)$ 、 苯环骨架振动 $\left(1619 、 1047 、 824 \mathrm{~cm}^{-1}\right.$ )及苯环上 $\mathrm{C}-\mathrm{H}$ 振动 $\left(745 \mathrm{~cm}^{-1}\right)$ 等对应 6 FAPB-CBDA 型聚酰亚胺分 子结构的特征峰。同时, 还可见 ZSM-5 中的 $\mathrm{Si}-\mathrm{O}$ 与 $\mathrm{Al}-\mathrm{O}\left(795 \mathrm{~cm}^{-1}\right)$ 对称伸缩振动峰 ${ }^{[19]}$ 。

从图 2 还可以看出, 在炭膜谱图中酰亚胺特征

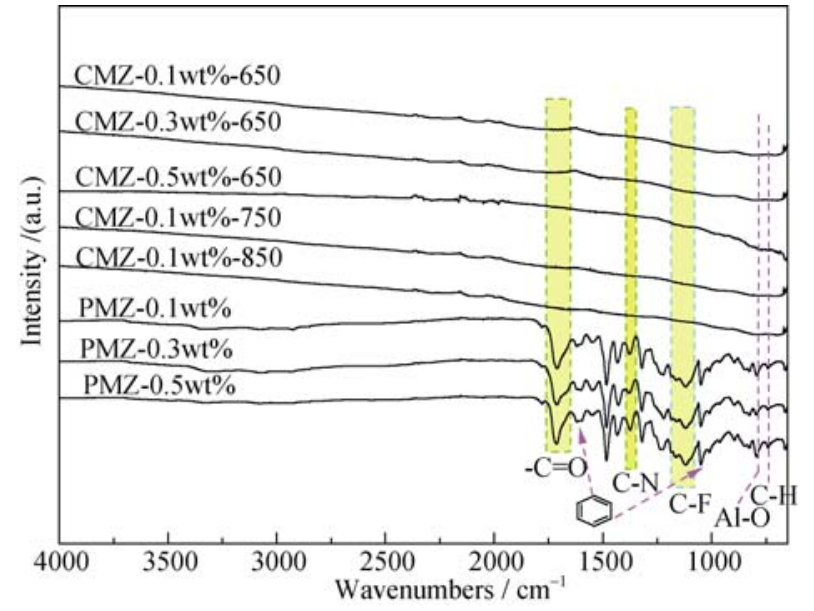

图 2 样品的红外光谱图

Fig. 2 Infrared spectra of samples

峰已完全消失, 表明聚酰亚胺中主要官能团已分解 脱落, 残留基体经缩聚与重排反应演变为类石墨化 稠环结构。此外, ZSM-5 的特征峰也明显减弱甚至 消失, 可能是由于热解后沸石表面被炭质基体严密 包裹, 导致衍射峰强度微弱。因此, 由沸石引起的炭 膜微观结构变化势必对其分离性能产生一定程度影 响，以此达到调节分离性目的。

\section{3 微结构分析}

通过 XRD 谱图(图 3)对炭膜的微观结构进行了 分析。从图 3(a) 可知所有前驱体膜均在 $2 \theta=10^{\circ} \sim 30^{\circ}$ 间存在一个较宽衍射峰。当引入 ZSM-5 后, 峰强度 降低, 衍射角度向高峰位偏移。由图 3(a)还可知, 随 着沸石含量增加, 膜材料微结构参数 $d_{002}$ 值越小, 表明添加沸石可以提高前驱体密度。另外, 当沸石 含量较高时 (即 PMZ-0.3wt\%和 PMZ-0.5wt\%), 在 $2 \theta=7.9^{\circ} 、 8.9^{\circ}$ 及 $23.1^{\circ}$ 处出现较弱的 ZSM-5 特征峰 ${ }^{[20]}$ 。 从图 3(b) 可以看见炭材料在 $2 \theta=22^{\circ}$ 的(002)面和 $44^{\circ}$ 的(100)面衍射峰。另外, 随着 ZSM-5 掺杂量增加, 虽 然(002)面衍射角度略发生偏移, 但峰形状显著改变, 表明微观结构发生变化。从图 3(c)可知, 随着热解温 度升高, 炭膜(002)和(100)面衍射角度增大, $d_{002}$ 值递 减, 说明高温使微结构石墨化程度提高, 趋于致密。

\section{4 炭膜微观形貌}

图 4 给出了炭膜的 SEM 照片, 可知炭膜表面平 整致密、无缺陷。而从断面插图可以看出, 分离层 与支撑体间结合紧密，分离层非常致密，而支撑体 孔隙发达。对比可知, 经沸石杂化炭膜的分离层厚 度较大, 这是由于引入的 ZSM-5 降低了前驱体成膜 液粘度和流动性所致。另外, ZSM-5 均匀地分布在 炭膜表层里，无显著团聚现象。 

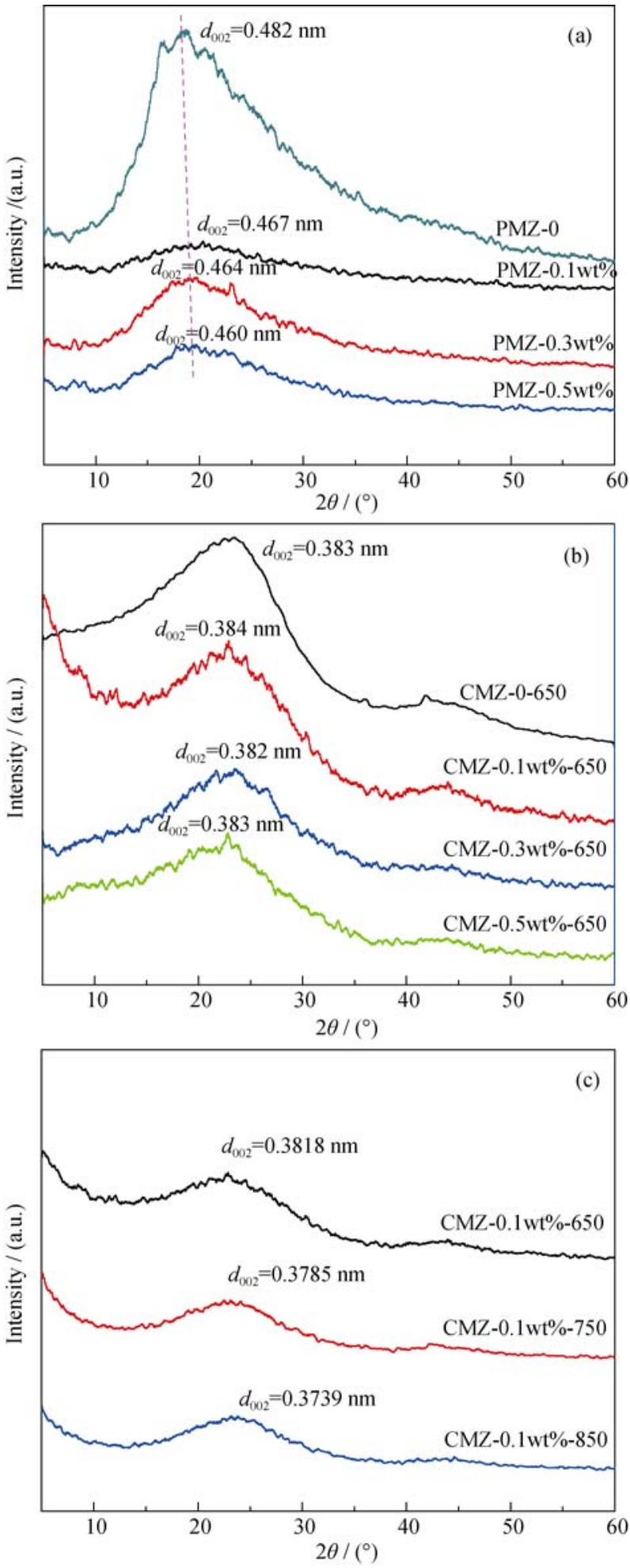

图 3 不同膜样品的 XRD 图谱

Fig. 3 XRD patterns of different samples

(a) Precursor membranes; (b) Hybrid carbon membranes; (c) Influence of pyrolysis temperature

\section{5 炭膜的分离性能}

表 1 给出了炭膜的气体分离性数据。可知, 所 有炭膜的气体渗透性遵循 $\mathrm{H}_{2}>\mathrm{CO}_{2}>\mathrm{N}_{2}$, 与气体分 子直径顺序相反, 表明均以分子篮分机理为主导。
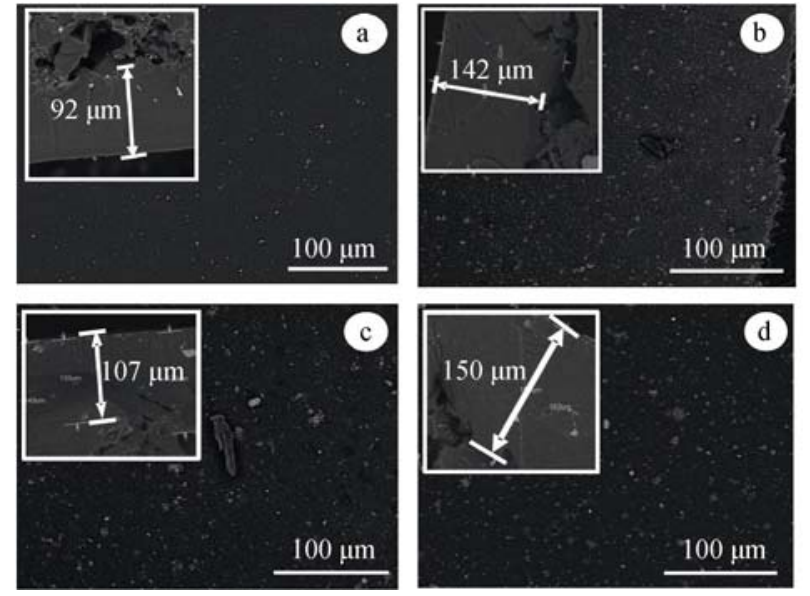

图 4 炭膜的 SEM 照片

Fig. 4 SEM images of carbon membranes

(a) CMZ-0-650; (b) CMZ-0.1wt\%-650; (c) CMZ-0.3wt\%-650; (d) CMZ-0.5wt $\%-650$

另外，表 1 数据还说明通过旋涂法易于在炭质支撑 体上制备高分离性炭膜，从而克服了陶瓷、不锈钢、 硅等支撑体成膜性差、易产生缺陷等问题 ${ }^{[21]}$ 。

在炭膜中掺杂沸石后，气体渗透性与选择性显 著提高了 1 2 个数量级。这是因为沸石自身丰富孔 隙结构为炭膜提供了大量通道, 有利于降低气体扩 散阻力。随着 ZSM-5 含量增加, 炭膜的渗透性先降 低后增大、选择性有所降低。此过程机理可借助图 5 加以解释, 即基体内沸石对炭膜气体渗透性的影 响体现在两个微区间的贡献：因沸石催化作用在其 周围的致密相界面结构层 ${ }^{[24]}$, 以及沸石内部的丰富 孔隙。当杂化炭膜中沸石含量较低时, 较薄的相界 面层对渗透性阻力与尺度较大的多孔沸石颗粒对渗 透性的促进贡献几乎可以忽略，从而提高渗透性; 当沸石含量适中时，随着炭膜基体内致密相界面层 增多, 气体渗透阻力增大, 渗透性降低; 而当沸石 含量较高时，不仅沸石颗粒相连，破坏了致密相界 面，而且也会形成颗粒间空隙，减小渗透阻力，同 时沸石的吸附扩散效应对气体渗透的贡献更显著, 从而提高渗透性 ${ }^{[25]}$ 。

另外, 随着热解温度的升高, 炭膜微结构经重 排缩聚反应，石墨化程度更高，结构更趋于致密， 并发生孔隙合并与塌陷现象 ${ }^{[26]}$, 从而使渗透性与选 择性均降低。为了综合评价炭膜的气体分离性能, 将 $\mathrm{H}_{2} / \mathrm{N}_{2}$ 分离性数据绘制成 Robeson 图(图 6)。本实 验制作的炭膜渗透性均优于文献中所报道的聚酰亚 胺基炭膜 ${ }^{[22-23]}$, 且许多数据点已落在极具商业吸引 力的范围, 表明 6FAPB-CBDA型聚酰亚胺是良好的 炭膜前驱体材料。特别是三个经 $650^{\circ} \mathrm{C}$ 热解得到的 杂化炭膜均已明显超越 Robeson 在 2008 年提出的分 
表 1 炭膜的气体分离性能数据

Table 1 Data of gas separation performance of carbon membranes

\begin{tabular}{|c|c|c|c|c|c|}
\hline \multirow{2}{*}{ Sample codes } & \multicolumn{3}{|c|}{ Permeability /Barrer ${ }^{a}$} & \multicolumn{2}{|c|}{ Selectivity } \\
\hline & $\mathrm{H}_{2}$ & $\mathrm{CO}_{2}$ & $\mathrm{~N}_{2}$ & $\mathrm{H}_{2} / \mathrm{N}_{2}$ & $\mathrm{H}_{2} / \mathrm{CO}_{2}$ \\
\hline CMZ-0-650 & 47.2 & 41.9 & 3.6 & 13.1 & 1.1 \\
\hline CMZ-0.1wt\%-650 & 1474.6 & 324.6 & 15.7 & 93.9 & 4.6 \\
\hline CMZ-0.1wt\%-750 & 119.4 & 48.2 & 4.1 & 29.1 & 2.5 \\
\hline CMZ-0.1wt\%-850 & 51.8 & 37.9 & 2.7 & 19.1 & 1.4 \\
\hline CMZ-0.3wt\%-650 & 776.6 & 96.5 & 11.0 & 70.5 & 8.0 \\
\hline CMZ-0.5wt\%-650 & 1029.1 & 102.3 & 15.3 & 67.2 & 10.0 \\
\hline$[22]$ & 628.0 & 302.0 & 38.6 & 16.2 & 2.1 \\
\hline$[23]$ & 298.1 & 106.8 & 48.9 & 6.1 & 2.8 \\
\hline$[22]$ & 308.0 & -- & -- & 2.0 & -- \\
\hline
\end{tabular}

Note: ${ }^{\text {a }} 1$ Barrer $=1 \times 10^{-10} \mathrm{~cm}^{3}(\mathrm{STP}) \cdot \mathrm{cm} /\left(\mathrm{cm}^{2} \cdot \mathrm{s} \cdot \mathrm{cm} \mathrm{Hg}\right)=7.5 \times 10^{-5} \mathrm{~cm}^{3}(\mathrm{STP}) \cdot \mathrm{cm} /\left(\mathrm{cm}^{2} \cdot \mathrm{s} \cdot \mathrm{kPa}\right)$
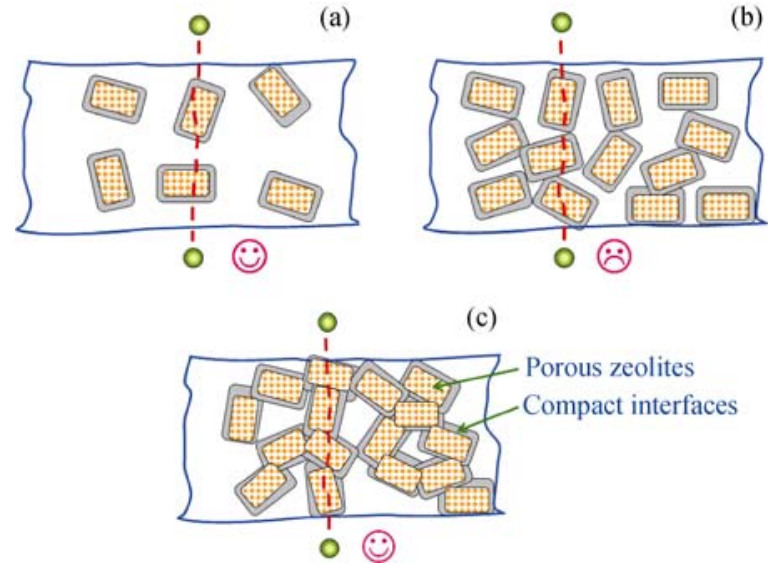

图 5 沸石含量对杂化炭膜渗透性影响示意图

Fig. 5 Schematic of the effect of zeolite content on the permeability for hybrid carbon membranes

(a) Low content; (b) Moderate content; (c) High content

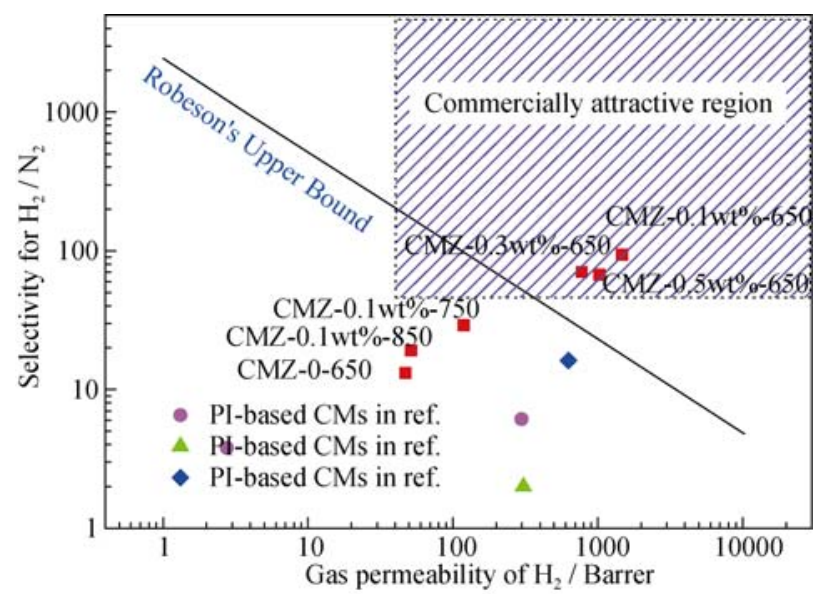

图 6 炭膜 $\mathrm{H}_{2} / \mathrm{N}_{2}$ 分离性的 Robeson 图

Fig. 6 Robeson's plot of carbon membranes for the separation performance of $\mathrm{H}_{2} / \mathrm{N}_{2}$

离性能上界限，表明沸石有效地改善了炭膜对氢气 的渗透性与 $\mathrm{H}_{2} / \mathrm{N}_{2} 、 \mathrm{H}_{2} / \mathrm{CO}_{2}$ 选择性, 显示出其在
IGCC 与燃料电池等方面潜在应用前景。

\section{3 结论}

以 ZSM-5 型沸石为掺杂剂、6FAPB-CBDA 型 聚酰亚胺为前驱体，经旋涂法成功制备了支撑炭 膜。沸石的引入在炭膜基体中构造了更多有利于气 体渗透的通道，从而改善了炭膜微结构与气体分离 性能。随着 ZSM-5 含量增加, 炭膜的渗透性先降低后 增大、选择性也有所降低。随着热解温度的升高, 炭膜 的渗透性与选择性均降低。当沸石含量为 $0.1 \mathrm{wt} \%$ ，热 解温度为 $650^{\circ} \mathrm{C}$ 时, 炭膜对 $\mathrm{H}_{2}$ 的渗透性为 1474.6 Barrer, 选择性达到 $93.9\left(\mathrm{H}_{2} / \mathrm{N}_{2}\right)$ 与 $4.6\left(\mathrm{H}_{2} / \mathrm{CO}_{2}\right)$ 。

\section{参考文献:}

[1] ABEDINI R, OMIDKHAH M, DOROSTI F. Hydrogen separation and purification with poly (4-methyl-1-pentyne)/MIL 53 mixed matrix membrane based on reverse selectivity. Int. J. Hydrogen Energ., 2014, 39(15): 7897-7909.

[2] ESCOLASTICO S, SOLIS C, SERRA J M. Hydrogen separation and stability study of ceramic membranes based on the system

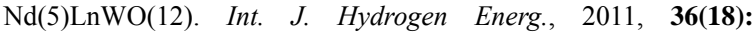
11946-11954.

[3] LEWIS A E, ZHAO H B, SYED H, et al. PdAu and PdAuAg composite membranes for hydrogen separation from synthetic watergas shift streams containing hydrogen sulfide. J. Membr. Sci., 2014, 465: $167-176$

[4] YUN S, OYAMA S T. Correlations in palladium membranes for hydrogen separation: a review. J. Membr. Sci., 2011, 375(1/2): $28-45$.

[5] SALLEH W N W, ISMAIL A F, MATSUURA T, et al. Precursor selection and process conditions in the preparation of carbon 
membrane for gas separation: a review. Sep. Purif. Rev., 2011, 40(4): 261-311.

[6] SAUFI S M, ISMAIL A F. Fabrication of carbon membranes for gas separation - a review. Carbon, 2004, 42(2): 241-259.

[7] ISMAIL A F, DAVID L I B. A review on the latest development of carbon membranes for gas separation. J. Membr. Sci., 2001, 193(1): $1-18$.

[8] CHEUNG O, HEDIN N. Zeolites and related sorbents with narrow pores for $\mathrm{CO}_{2}$ separation from flue gas. $R S C A d v$, 2014, 4(28): 14480-14494.

[9] PAUL D R. Creating new types of carbon-based membranes. Science, 2012, 335(6067): 413-414.

[10] TIN P S, XIAO Y, CHUNG T S. Polyimide - carbonized membranes for gas separation: structural, composition, and morphological control of precursors. Sep. Purif Rev., 2006, 35(4): $285-318$

[11] ZHANG X, HU H, ZHU Y, et al. Carbon molecular sieve membranes derived from phenol formaldehyde novolac resin blended with poly(ethylene glycol). J. Membr. Sci., 2007, 289(1/2): 86-91.

[12] KABURAGI Y, HISHIYAMA Y, OKA H, et al. Growth of iron clusters and change of magnetic property with carbonization of aromatic polyimide film containing iron complex. Carbon, 2001, 39(4): 593-603.

[13] ZHANG X, LIU H, WANG T, et al. Modification of carbon membranes and preparation of carbon-zeolite composite membranes with zeolite growth. Carbon, 2006, 44(3): 501-507.

[14] TEIXEIRA M, RODRIGUES S C, CAMPO M, et al. Boehmitephenolic resin carbon molecular sieve membranes- permeation and adsorption studies. Chem. Eng. Res. Des., 2014, 92(11): 2668-2680.

[15] ZHANG B, WANG T, WU Y, et al. Preparation and gas permeation of composite carbon membranes from poly(phthalazinone ether sulfone ketone). Sep. Purif. Technol., 2008, 60(3): 259-263.

[16] ZHANG B, SHI Y, WU Y, et al. Preparation and characterization of supported ordered nanoporous carbon membranes for gas sepa- ration. J. Appl. Polym. Sci., 2014, 131(4): 2136-2146.

[17] LI Y, CHUNG T S. Exploratory development of dual-layer carbonzeolite nanocomposite hollow fiber membranes with high performance for oxygen enrichment and natural gas separation. Micropor. Mesopor. Mat., 2008, 113(1/2/3): 315-324.

[18] ZHANG B, SHI Y, WU Y H, et al. Towards the preparation of ordered mesoporous carbon/carbon composite membranes for gas separation. Sep. Sci. Technol., 2014, 49(2): 171-178.

[19] LUA A C, SHEN Y. Preparation and characterization of polyimidesilica composite membranes and their derived carbon-silica composite membranes for gas separation. Chem. Eng. J., 2013, 220: $441-451$.

[20] ZHANG B, WU Y, LU Y, et al. Preparation and characterization of carbon and carbon/zeolite membranes from ODPA-ODA type polyetherimide. J. Membr. Sci., 2015, 474: 114-121.

[21] MA X L, LIN B K, WEI X T, et al. Gamma-alumina supported carbon molecular sieve membrane for propylene/propane separation. Ind. Eng. Chem. Res., 2013, 52(11): 4297-4305.

[22] ITTA A K, TSENG H H, WEY M Y. Effect of dry/wet-phase inversion method on fabricating polyetherimide-derived CMS membrane for $\mathrm{H}_{2} / \mathrm{N}_{2}$ separation. Int. J. Hydrogen Energ., 2010, 35(4): $1650-1658$

[23] TSENG H H, SHIU P T, LIN Y S. Effect of mesoporous silica modification on the structure of hybrid carbon membrane for hydrogen separation. Int. J. Hydrogen Energ., 2011, 36(23): $15352-15363$

[24] TIN P S, CHUNG T S, JIANG L, et al. Carbon-zeolite composite membranes for gas separation. Carbon, 2005, 43(9): 2025-2027.

[25] LIU Q, WANG T, GUO H, et al. Controlled synthesis of high performance carbon/zeolite $\mathrm{T}$ composite membrane materials for gas separation. Micropor. Mesopor. Mat., 2009, 120(3): 460-466.

[26] WANG T, ZHANG B, QIU J, et al. Effects of sulfone/ketone in poly(phthalazinone ether sulfone ketone) on the gas permeation of their derived carbon membranes. J. Membr. Sci., 2009, 330(1/2): 319-325. 